\title{
Off-axis directional beaming via photonic crystal surface modes
}

Cite as: Appl. Phys. Lett. 92, 092114 (2008); https://doi.org/10.1063/1.2842424

Submitted: 17 October 2007 . Accepted: 21 January 2008 . Published Online: 07 March 2008

Humeyra Caglayan, Irfan Bulu, and Ekmel Ozbay

\section{ARTICLES YOU MAY BE INTERESTED IN}

Off-axis beaming from subwavelength apertures

Journal of Applied Physics 104, 073108 (2008); https://doi.org/10.1063/1.2990063

Off-axis directional beaming of optical field diffracted by a single subwavelength metal slit with asymmetric dielectric surface gratings

Applied Physics Letters 90, 051113 (2007); https://doi.org/10.1063/1.2437730

Enhanced transmission and beaming via a zero-index photonic crystal

Applied Physics Letters 109, 031105 (2016); https://doi.org/10.1063/1.4959085

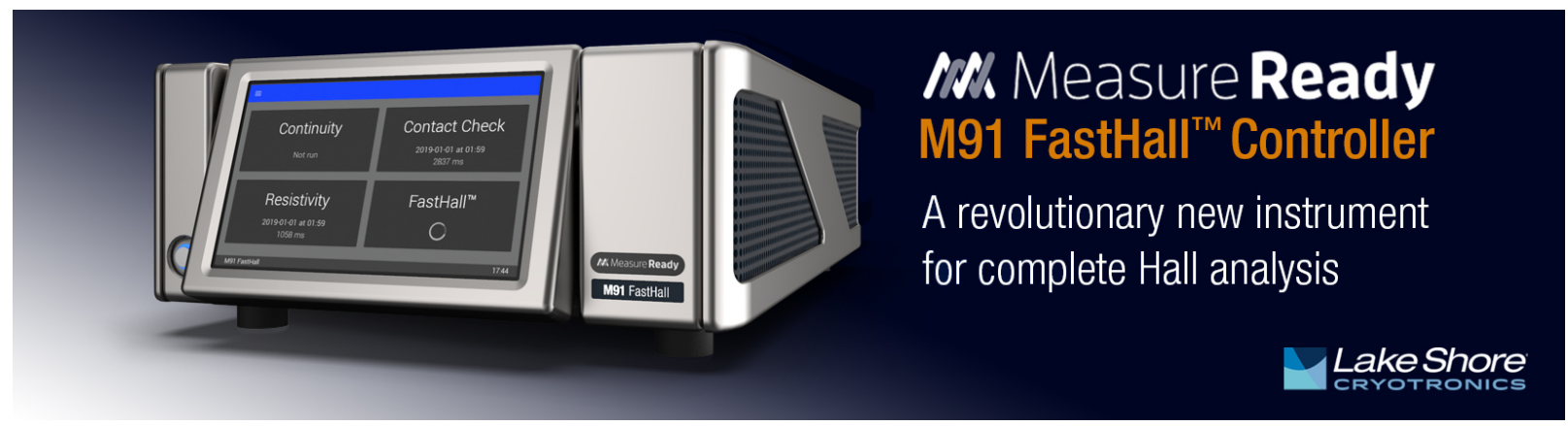




\title{
Off-axis directional beaming via photonic crystal surface modes
}

\author{
Humeyra Caglayan, ${ }^{1, a)}$ Irfan Bulu, ${ }^{2}$ and Ekmel Ozbay ${ }^{1}$ \\ ${ }^{1}$ Nanotechnology Research Center-NANOTAM, Department of Physics, Department of Electrical \\ and Electronics Engineering, Bilkent, 06800 Ankara, Turkey \\ ${ }^{2}$ School of Engineering and Applied Sciences, Harvard University, 29 Oxford Street, Cambridge, \\ Massachusetts 02138, USA
}

(Received 17 October 2007; accepted 21 January 2008; published online 7 March 2008)

\begin{abstract}
We investigated off-axis directional beaming from a photonic crystal (PC) waveguide. We theoretically and experimentally demonstrated that it is possible to control beaming from a PC waveguide via PC surface modes using a gratinglike layer with different periods on different sides of the waveguide. We observed off-axis directional beaming with full width at half maximum of $10^{\circ}$ with a beaming angle of $14^{\circ}$ from a PC waveguide. Since the beaming angle only depends on the periods of the gratinglike layer on the output side, this beaming angle can be easily changed by arranging the grating periods asymmetrically. (c) 2008 American Institute of Physics.
\end{abstract}

[DOI: $10.1063 / 1.2842424$ ]

The diffraction limit is the main problem in manipulating light, especially for subwavelength scales. Electromagnetic (EM) waves that transmit through a subwavelength aperture are fully diffracted in all directions, as defined in the standard diffraction theory by Bethe ${ }^{1}$ in 1944. In order to overcome this disadvantage, researchers have studied beaming phenomena for many years now. In 2002, Lezec et al. ${ }^{2}$ showed that it is possible to obtain directional beaming from subwavelength apertures via the excitation of surface plasmons (SPs) on corrugated metallic surfaces. The SPs that scatter from the output surface produce emitters that are similar to an array antenna. Hence, the constructive interference of the surface waves result in directional beaming. Since the reporting of directional beaming through subwavelength apertures, this phenomenon has been intensively investigated as per the theoretical and experimental aspects. ${ }^{3-5}$

Since the arrival of photonic crystals ${ }^{6,7}(\mathrm{PCs})$, many applications based on PCs have been proposed and demonstrated. Waveguides ${ }^{8}$ and highly directional antennae $e^{9,10}$ are among these applications. PCs also have another important property that can lead to important applications. PCs can support surface propagating EM waves for the appropriate crystal termination surfaces. ${ }^{11,12}$ Therefore, the surface of a corrugated PC has a similar property as corrugated metallic surfaces. A PC waveguide which has a width that is less than the wavelength, also suffers from a diffraction limit. Hence, this similarity has been suggested in order to solve this problem and by using this similarity it has been shown that it is possible to obtain directional beaming from a PC waveguide. $^{13,14}$

On the other hand, one needs to control directional beaming and the direction of the beaming in order to fully solve the problem of diffraction from a subwavelength aperture. Since controlling the direction of beaming is another important issue, all of these studies are specifically investigated on-axis beaming. Recently, Kim et al. ${ }^{15}$ proposed a method for off-axis directional beaming and Lin et al. ${ }^{16}$ showed that it is possible to control beaming at optical frequencies using asymmetric gratings. However, there has not been any conducted work that demonstrates off-axis direc-

\footnotetext{
${ }^{a)}$ Electronic mail: caglayan@fen.bilkent.edu.tr.
}

tional beaming via the surface modes of a PC. In the present paper, we investigated the control of directional beaming via a PC waveguide with asymmetric surface corrugations. By using this new structure, we were able to change the beaming angle and attain off-axis directional beaming from a PC waveguide with a subwavelength width.

There are three types of modes that are supported by a finite size PC, which is finite in one dimension and infinitely periodic in the other dimension. The first of these modes extends in the air and PC. The second mode extends in the air but decays in the PC and the last mode decays in the air but extends in the PC. On the other hand, the PC can also support one more mode by modifying the PC-air interface. This mode is known as the surface propagating mode, the electric field of this mode decays in the air and PC. The electric field of this mode is localized at the surface of the PC. There are several ways to create this mode such as using rods of different shapes or using rods with a different radius at the surface. In the present work, we reduced the radius of the rods at the surface of the PC.

We started with a two-dimensional PC constructed from a $43 \times 11$ square array of circular alumina rods. The crystal is 11 layers along the propagation direction. The radius of the rods is $1.55 \mathrm{~mm}$ and the dielectric constant of the alumina is 9.61. The separation between the center of the rods along lattice the vectors is $a=11 \mathrm{~mm}$. This PC has a band gap between 8.7 and $13.2 \mathrm{GHz}$. Then, we removed one row of rods from this PC in order to obtain a waveguide. We also modified the surface by adding a layer of rods with a radius of $0.76 \mathrm{~mm}$ to this PC waveguide to create surface propagating modes in the band gap. Figure 1 shows the calculated field intensity by the finite-difference time-domain (FDTD) method when the surface corrugation is added to the exit surface of the PC waveguide at $11.1 \mathrm{GHz}$. The data show that the surface modes are not coupled to the radiating modes of the free space. However, the surface modes excited by the PC waveguide can be coupled to the radiating modes of the free space when an extra layer, called a gratinglike layer, is added in front of the corrugated layer of the PC.

The PC waveguide used in our study has a width that is smaller than the wavelength radiation from the waveguide would diffract in all directions. Directional beaming along 


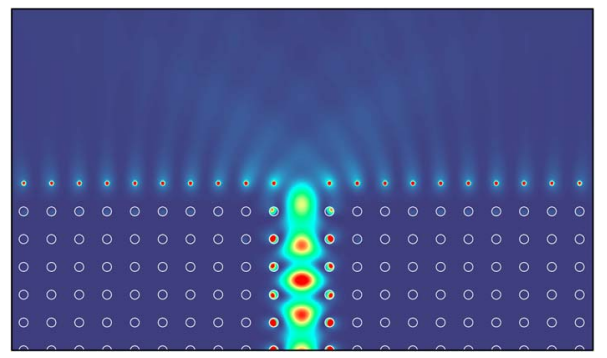

FIG. 1. (Color online) Calculated field intensity when the surface corrugation is added to the exit surface of the PC waveguide.

the axis of the propagating direction from the PC waveguide was observed by adding a corrugated layer and a gratinglike layer with a period of $22 \mathrm{~mm}$ in front of the PC waveguide. ${ }^{14}$ In the present study, in order to obtain off-axis directional beaming from a PC waveguide, we used gratinglike layers with different periods on different sides of the output surface of the waveguide.

The experimental setup consists of a network analyzer and two standard-gain horn antennae in order to measure the transmission amplitude between 10 and $13 \mathrm{GHz}$. Radiation is normally incident upon the PC from $15 \mathrm{~cm}$ by the source antenna. The receiver antenna is $100 \mathrm{~cm}(\approx 50 \lambda)$ away from the PC's back face, and was connected to a rotating arm in order to measure the angular dependence of the far-field radiation. The schematic of the experimental setup and PC waveguide is illustrated in Fig. 2. We formed the gratinglike layer with rods that have equal radii of the bulk PC rods. The asymmetric gratinglike layer has a double period $(22 \mathrm{~mm})$ on one side and triple period $(33 \mathrm{~mm})$ on the other side of the PC waveguide (Fig. 2).

The measured and calculated radiation patterns of the EM waves emitted from the PC waveguide at $11.1 \mathrm{GHz}$ are shown in Fig. 3. We observed a beam that was confined to a narrow angular region with a FWHM of $10^{\circ}$ and a beaming angle of $14^{\circ}$ (Fig. 3). This result is in good agreement with the FDTD calculations. In addition, we measured the electric field intensity at $11.1 \mathrm{GHz}$ over a region of $60 \times 70 \mathrm{~cm}^{2}$. Figure 4 shows that the electric field intensity is confined to

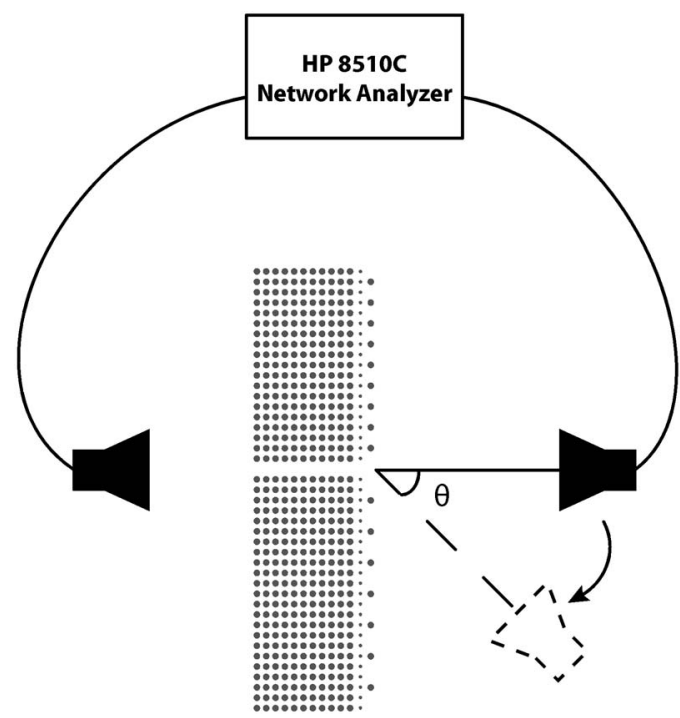

FIG. 2. Experimental setup for angular distribution measurements. $\Theta$ changes from $0^{\circ}$ to $180^{\circ}$. The right side of the $90^{\circ}$ stands for the side with a gratinglike layer period of $33 \mathrm{~mm}$.

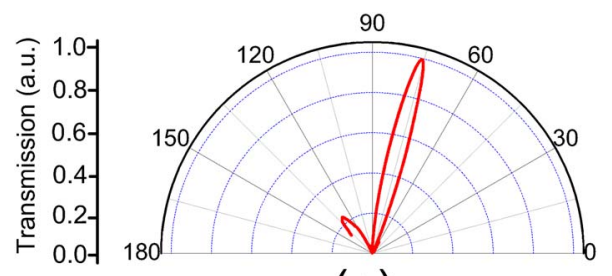

(a)

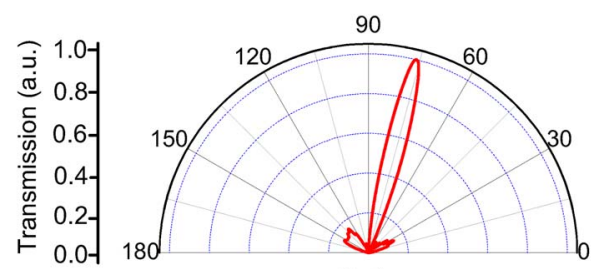

(b)

FIG. 3. (Color online) The measured (a) and calculated (b) radiation patterns of the EM waves emitted from the PC waveguide at $11.1 \mathrm{GHz}$. The right side of $90^{\circ}$ stands for the side with a gratinglike layer period of $33 \mathrm{~mm}$.

a narrow spatial region and propagates along $14^{\circ}$ without diffracting. Hence, the asymmetric gratinglike layers provide an off-axis directional beaming from the PC waveguide via PC surface modes.

The off-axis beaming can be explained using the SP diffraction theory. This theory states that when a wave vector of the SP is smaller than the grating vector, the wave vector of the diffracted light will be negative, or vice versa. ${ }^{3}$ This can also be stated as the projected direction of the diffracted beam is the opposite of the SP propagation when a wave vector of the SP is smaller than the grating vector. Since PC surface can be designed to support surface waves as described in the above, the same phenomenon holds for the surface waves of the PC, depending on the period of the gratinglike layer, the direction of the emitted beam changes. For directional beaming, the gratinglike layers with the same periods on both sides of the waveguide, the two emerging beams overlap to form a beaming pattern along the axis of propagation direction. On the other hand, the gratinglike lay-

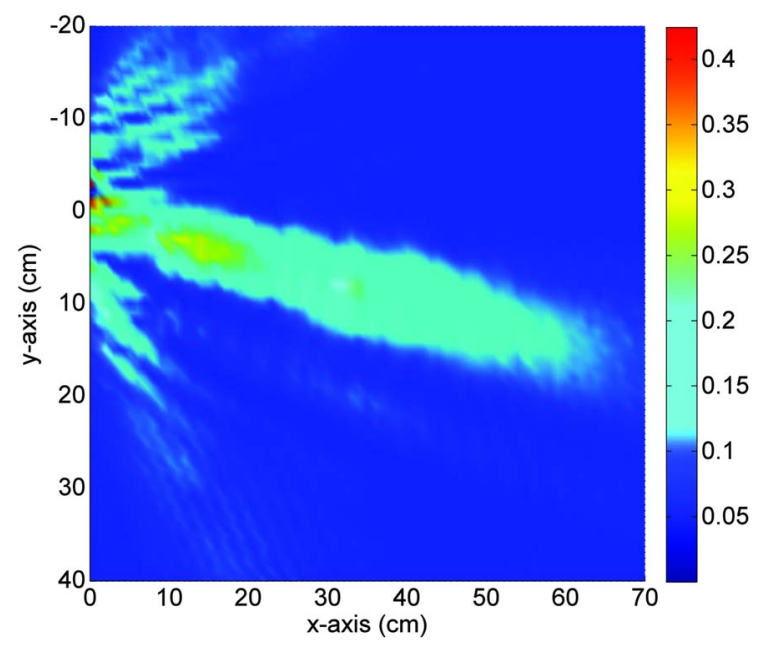

FIG. 4. (Color online) The measured intensity distribution at the exit side of the PC waveguide when the corrugation and gratinglike layers are added to the exit surface of the PC waveguide. The $Y$ axis is parallel to the PC surface and the positive side of the axis indicates the side of gratinglike layer with a period of $33 \mathrm{~mm}$. 
ers with different periods on different sides of the waveguide can be arranged such that the wave vector of one of the gratinglike layer is negative and the wave vector of the other gratinglike layer is positive. Hence, such a combination will provide a directional, yet off-axis, beaming. Therefore, the desired emerging beam angle can be achieved by arranging the grating periods of the output surface of the PC.

In conclusion, we were able to control the beaming angle of the directional beaming from a PC waveguide. Using a PC with a waveguide at the center and adding a corrugated surface, we were able to achieve surface modes. Then, we added an extra layer called a gratinglike layer, which has different periods on different sides of the PC waveguide. This gratinglike layer enables surface modes that are excited by a PC waveguide to couple the radiating modes of the free space. We showed that when using a gratinglike layer with different periods on different sides of the waveguide, it is possible to obtain off-axis directional beaming from a PC waveguide via PC surface modes. Moreover, the beaming angle only depends on the periods of the gratinglike layer on the output side. Therefore, this beaming angle can be easily changed by arranging the grating periods asymmetrically.

This work was supported by the European Union under the projects EU-NoE-METAMORPHOSE, EU-NoEPHOREMOST, and TUBITAK under Projects Nos.
104E090, 105E066, 105A005, and 106A017. One of the authors (E.O.) also acknowledges partial support from the Turkish Academy of Sciences.

${ }^{1}$ H. A. Bethe, Phys. Rev. 66, 163182 (1944).

${ }^{2}$ H. J. Lezec, A. Degiron, E. Devaux, R. A. Linke, L. Martin-Moreno, F. J. Garcia-Vidal, and T. W. Ebbesen, Science 297, 820 (2002).

${ }^{3}$ H. Caglayan, I. Bulu, and E. Ozbay, J. Opt. Soc. Am. B 23, 419 (2006).

${ }^{4}$ F. J. Garcia-Vidal, L. Martin-Moreno, H. J. Lezec, and T. W. Ebbesen, Appl. Phys. Lett. 83, 4500 (2003).

${ }^{5}$ S. S. Akarca-Biyikli, I. Bulu, and E. Ozbay, J. Opt. A, Pure Appl. Opt. 7, 159 (2005).

${ }^{6}$ E. Yablonovitch, Phys. Rev. Lett. 58, 2059 (1987).

${ }^{7}$ S. John, Phys. Rev. Lett. 58, 2486 (1987).

${ }^{8}$ S. G. Johnson, P. R. Villeneuve, S. Fan, and J. D. Joannopoulos, Phys. Rev. B 62, 8212 (2000).

${ }^{9}$ I. Bulu, H. Caglayan, and E. Ozbay, Appl. Phys. Lett. 83, 3263 (2003).

${ }^{10}$ S. Enoch, B. Gralak, and G. Tayeb, Appl. Phys. Lett. 81, 1588 (2002).

${ }^{11}$ R. D. Meade, K. D. Brommer, A. M. Rappe, and J. D. Joannopoulos, Phys. Rev. B 44, 10961 (1991).

${ }^{12}$ P. Kramper, M. Agio, C. M. Soukoulis, A. Birner, F. Muller, R. B. Wehrspohn, U. Gosele, and V. Sandoghdar, Phys. Rev. Lett. 92, 113903 (2004).

${ }^{13}$ E. Moreno, F. J. Garcia-Vidal, and L. Martin-Moreno, Phys. Rev. B 69, 121402 (2004).

${ }^{14}$ I. Bulu, H. Caglayan, and E. Ozbay, Opt. Lett. 30, 3078 (2005).

${ }^{15}$ S. Kim, H. Kim, Y. Lim, and B. Lee, Appl. Phys. Lett. 90, 051113 (2007).

${ }^{16}$ D.-Z. Lin, T.-D. Cheng, C.-K. Chang, J.-T. Yeh, J.-M. Liu, C.-S. Yeh, and C.-K. Lee, Opt. Express 15, 2585 (2007). 